\title{
Mimicry cannot explain rejection type in a host-brood parasite system
}

\author{
Michal Šulc ${ }^{1}$, Jolyon Troscianko ${ }^{2}$, Gabriela Štětková ${ }^{1,3}$, Anna \\ E. Hughes ${ }^{2}$, Václav Jelínek ${ }^{1}$, Miroslav
} Capek ${ }^{1}$ and Marcel Honza ${ }^{1}$

${ }^{1}$ Institute of Vertebrate Biology of the Czech Academy of Sciences, Květná 8, 603 65 Brno, Czech Republic

${ }^{2}$ Centre for Ecology \& Conservation, University of Exeter, Penryn, UK

${ }^{3}$ Department of Botany and Zoology, Faculty of Science, Masaryk University, Brno, Czech Republic

Author for correspondence: Michal Šulc, e-mail: sulc-michal@seznam.cz

\begin{abstract}
One of the most effective defensive strategies of hosts against brood parasites is rejection, commonly achieved by ejection of the parasitic egg or desertion of the parasitized nest. Nest desertion should be a costlier strategy than egg ejection because birds then must spend additional time and energy re-nesting, and therefore we still cannot explain why some individuals desert their nests rather than eject parasitic eggs and continue a given breeding attempt. The great reed warbler (Acrocephalus arundinaceus) is a frequent host of the common cuckoo (Cuculus canorus) and is known to use both types of rejection response. We investigated the hypothesis that the hosts desert if they cannot reliably recognize the cuckoo egg in their nest by measuring cuckoo egg mimicry. We predicted that we would find better mimicry when hosts deserted rather than ejected. However, we did not find a difference in mimicry between these two groups of nests, implying that host females do not desert because they cannot reliably recognize the parasitic egg. We also showed that neither the date in the season nor the age of the host females influences the type of the rejection. Other factors potentially eliciting nest desertion, including host personality, host, inability to eject, excessive clutch reduction and visibility of the cuckoo female at the host nest are discussed. Finally, we suggest that desertion may persist as a host defensive strategy against brood parasitism because it is not as costly as previously assumed and/or it is beneficial for host females in good physical condition.
\end{abstract}

Keywords: Brood parasitism, common cuckoo, great reed warbler, egg ejection, nest desertion, spectrometry, reflectance, colour contrast, Mica toolbox, pattern energy 


\section{Introduction}

It is surprisingly common to find seemingly abandoned bird nests. Parental mortality is a likely explanation in some cases, however, relatively few studies have shown that this is the main reason (e.g. Roche, Arnold, \& Cuthbert, 2010). Another explanation of nest abandonment, which seems to be much more common in nature, is predation of the nest content. It is well documented that clutch or brood reduction elicit abandonment not only in birds (Armstrong \& Robertson, 1988; Kosciuch, Parker, \& Sandercock, 2006; Moskát et al., 2011; Verboven \& Tinbergen, 2002) but also in insects (Zink, 2003) and fishes (Jennions \& Polakow, 2001; Zuckerman, Philipp, \& Suski, 2014). It has also been reported that even only potential risk of predation can lead in some bird species to nest desertion (Beckmann \& Martin, 2016; Flegeltaub, Biro, \& Beckmann, 2017).

Desertion behaviour has frequently been studied in the hosts of brood parasites as a defensive strategy against parasitism (Hanley et al., 2016). The parasite-host systems are particularly interesting because the hosts have an alternative and potentially less costly strategy: the ejection of the parasitic egg. However, some hosts use both of these strategies (Davies \& Brooke, 1989) despite the fact that nest desertion is presumably more costly than ejection, particularly when nests contain eggs due to the waste of energy and time invested into the nest building, egg laying and incubation (Rothstein, 1975; Servedio \& Hauber, 2006). Indeed, it was shown that even few days delay in the breeding caused by re-nesting incurs costs in terms of reduced clutch size (Øien, Moksnes, Røskaft, \& Honza, 1998) probably because the second breeding attempt may occur when environmental conditions are no longer optimal for reproduction (Immelmann, 1971; Rothstein, 1975). Apart from these direct costs of desertion, re-nesting also risks the possibility of being parasitized again and therefore may not necessarily free the hosts from parasitism (Rothstein, 1975). Why then is desertion still common in host species?

One reason why hosts may desert their nests is that brood parasites are also commonly nest predators (Arcese, Smith, \& Hatch, 1996; Davies, 2000; Gloag, Fiorini, Reboreda, \& Kacelnik, 2013; Øien et al., 1998) and nest desertion can be triggered simply by egg loss (Guigueno \& Sealy, 2010; Hill \& Sealy, 1994; Kosciuch et al., 2006; Moskát \& Honza, 2002; Moskát et al., 2011; Verboven \& Tinbergen, 2002). In this case, desertion may be seen as a rational response, as it does not pay to stay in nests with significantly reduced clutch if there is a chance to re-nest. However, it seems that host desertion behaviour can also be contemplated as an alternative response to parasitism (Hanley et al., 2016).

The other reason to desert the nest could simply be that hosts are physically unable to puncture or grasp and finally eject parasitic eggs (Rohwer \& Spaw, 1988). In support of this, nest desertion is often performed by small hosts like mangrove warblers (Setophaga petechia), chipping sparrows (Spizella passerina), meadow pipits (Anthus pratensis), blue-grey gnatcatchers (Polioptila caerulea) or Phylloscopus warblers that never or rarely eject parasitic eggs (Goguen \& Mathews, 1996; Guigueno \& Sealy, 2010; Martín-Vivaldi, Soler, Møller, Pérez-Contreras, \& Soler, 2013; Moksnes, Røskaft, \& Braa, 1991; Strausberger \& Hauber, 2017) while some medium and large-sized hosts e.g. blackbirds (Turdus merula), common grackles (Quiscalus quiscula) and Eurasian magpies (Pica pica) 
do not desert their nests and instead eject eggs as a response to parasitism (Hanley, Samaš, Hauber, \& Grim, 2015; Peer \& Rothstein, 2010; Soler, 1990; Soler, Ruiz-Raya, Roncalli, \& Ibáñez-Álamo, 2015), but see (Begum, Moksnes, Røskaft, \& Stokke, 2012). It has also been shown that some host species that are normally able to eject eggs of natural parameters in terms of size or eggshell thickness use nest desertion as an alternative response when they face abnormally large or hard experimental eggs (Honza \& Moskát, 2008; Stokke et al., 2010; Šulc, Procházka, Capek, \& Honza, 2015).

Physical incapacity is presumably not the only problem for parasitic egg ejection. For successful ejection of parasitic eggs, birds need also to recognize them, while nest desertion only requires suspecting that the nest has been parasitized. Therefore, it has been suggested that species may desert their nests because they have not evolved sufficient recognition abilities (Hosoi \& Rothstein, 2000; Sealy \& Lorenzana, 1997). However, there are many species that are able to recognize and eject parasitic eggs, but still desert their nests quite regularly (Davies \& Brooke, 1989).

Therefore, we might also expect to find within-species differences in nest abandonment as a response to highly mimetic parasitic eggs. Individual hosts that face a highly mimetic parasitic egg should be more likely to desert because the parasitic egg is difficult to identify, leading to a higher risk of recognition error (Davies \& Brooke, 1988; Molnár, 1944; Rodriguez-Girones \& Lotem, 1999; Stokke, Moksnes, \& Røskaft, 2005) and subsequent misdirected care of brood parasite. Thus, nest desertion may be a less costly strategy than unsuccessful ejection of parasitic egg. We hypothesize that if birds are able to assess the risk of recognition error, they should be able to induce nest desertion when this risk is high. According to this hypothesis we assume that deserting hosts know that their nest was parasitized. They can gain this information e.g. simply from observing the cuckoo female parasitizes their nest which happens in the majority of cases in our study host species (Jelínek et al. in prep) or because hosts may determine parasitism based on increased variance among eggs in their clutch (Hosoi and Rothstein 2000).

The great reed warbler (Acrocephalus arundinaceus, hereafter GRW) is an ideal study species for testing this hypothesis. It is heavily parasitized by the common cuckoo (hereafter cuckoo), GRW females are puncture-ejectors with well-developed recognition abilities (Bártol, Karcza, Moskát, Røskaft, \& Kisbenedek, 2002; Honza \& Moskát, 2008) and it seems that mimicry of parasitic eggs is the main factor triggering rejection behaviour (Bártol et al., 2002). GRW females reject cuckoo eggs by egg ejection or nest desertion at a relatively similar rate (Bártol et al., 2002; Moskát \& Honza, 2002), and it has been shown that they have recognition problems when faced with highly mimetic parasitic eggs, leading to a delayed response (Antonov, Stokke, Moksnes, \& Røskaft, 2007; Lotem, Nakamura, \& Zahavi, 1995) and significantly less intense rejection (pecking) behaviour (Antonov et al., 2007) indicating lower confidence during decision making (Ratcliff, Smith, Brown, \& McKoon, 2016) then when they respond to non-mimetic eggs. Using spectrometry and image analysis techniques we measured the similarity between cuckoo and host eggs (egg mimicry) in naturally parasitized GRW nests and predicted that GRW females should eject 
cuckoo eggs that poorly match their own eggs (with lower risk of recognition error) more often than highly mimetic cuckoo eggs (with higher risk of recognition error) that should instead be deserted.

\section{Methods}

Study system and data collection

All data were collected in the fishpond area between Mutěnice $\left(48^{\circ} 54^{\prime} \mathrm{N}, 17^{\circ} 02\right.$ 'E) and Hodonín $\left(48^{\circ} 51^{\prime} \mathrm{N}, 17^{\circ} 07^{\prime} \mathrm{E}\right)$, South Moravia, Czech Republic between 2013 and 2018. In this area, the host population of the GRW is long-term studied and all birds are individually marked. Most nests were found during the building stage when mapping male territories and their mating status (Bensch, 1996) and checked every day until the clutch completion and approximately every third day during incubation (see (Jelínek, Procházka, \& Honza, 2015) for detailed description of the study site).

The GRW population in this area experiences a cuckoo parasitism rate of about $69 \%$ ( $N=380$ of 549 nests, data from 2013 - 2018), of which $39 \%$ were parasitized multiple times (147 nests). Host rejection rate is about $57 \%$ in nests parasitized once ( $\mathrm{N}=124$ of 216 nests where we were able to determine host response) and we observed two types of rejection behaviour in the GRW; an ejection if the cuckoo egg disappeared from the nest during incubation (77 cases), or a nest desertion if the nest was abandoned by the GRW during incubation (47 cases). In multiply parasitized nests we recorded an even higher rejection rate (where at least one cuckoo egg was rejected by ejection or desertion) of about $64 \%(N=21$ of 33 nests where we were able to determine host response), of which $62 \%$ were deserted (13 nests). Only females of GRW incubate eggs and eject cuckoo eggs (Požgayová, Procházka, \& Honza, 2009), so we can expect that probably only females are responsible for the egg rejection.

For the purpose of the study, we only used nests that were parasitized once to be sure that hosts responded to a specific cuckoo egg. As clutch size reduction can also induce nest desertion (Kosciuch et al., 2006; Moskát et al., 2011; Verboven \& Tinbergen, 2002), we used nests where the overall clutch size was not reduced after parasitism; i.e. cuckoo females took either one or no host eggs during the parasitism act. We show that this difference did not influence type of host response (Fisher's exact test, 79 nests where cuckoo removed 1 host egg and 24 nests where cuckoo did not remove any eggs, of which 29 and four nests were deserted respectively; $P=0.082$ ). We also demonstrate that our nest visits did not elicit nest desertion because none of the randomly sampled nonparasitized nests visited at the same frequency were deserted $(N=26)$. A previous study from the same area has also shown that hosts desert their nests because of brood parasitism (Hanley et al., 2016). It was also shown that hosts parasitized later in the season tend to accept a parasitic egg more often than those parasitized earlier (Sealy, 1995). Therefore, we can also expect effect of timing on type of the host rejection, more specifically that nests parasitized earlier could be deserted more often because hosts should still have enough time for re-nesting, which may not be the case at the end of the breeding season. Finally, it was demonstrated that naïve GRW first-time breeders tend to accept cuckoo eggs perhaps because they cannot recognize their own reliably (Lotem, 
Nakamura, \& Zahavi, 1992) but see also (Amundsen, Brobakken, Moksnes, \& Røskaft, 2002; Grim, Samaš, \& Hauber, 2014; Procházka et al., 2014; Stokke, Rudolfsen, Moksnes, \& Røskaft, 2004), for opposite results). Therefore, it is also possible that younger GRW females may desert more often than experienced older females. Hence, we determined age of all GRW females. Some of them were born in the study area or nested here in previous years, and thus we knew their exact or minimum age $(\mathrm{N}=47)$. In the remaining cases $(\mathrm{N}=21)$ we used a scoring method combining the coloration of iris, tarsus and tongue (Bensch, Hasselquist, Nielsen, \& Hansson, 1998; Procházka, Jelínek, Požgayová, \& Honza, 2012) to discriminate between first time breeders and older birds. Then we divided all females into the two age groups, first time breeders in their second year of life and birds in their third year of life (at least) with possible breeding experience (Bensch et al., 1998; Procházka et al., 2012).

\section{Egg measurements and mimicry calculation}

Once we found a cuckoo egg in the GRW nest, we measured its background colour and size and took a photo of this egg (for details see below). For mimicry assessment, we repeated this procedure for all host eggs after the host female finished her laying.

The background colour of eggs was measured using a Jaz Spectrometer (Ocean Optics, Dunedin, FL) in the range 300 to $700 \mathrm{~nm}$ which is the range that birds can perceive (Cuthill, 2006). Overall, we took nine measurements (each covering ca. $1 \mathrm{~mm}^{2}$ ) at three different parts of egg (sharp pole, middle part, and blunt pole). Since we focused on background colour, we avoided measuring dark spots (Šulc, Procházka, Capek, \& Honza, 2016). For each egg we used the measurement with the highest reflectance that best corresponds to the colour of the background. To get one average colour of host eggs in the same clutch, we simply averaged the colour of individual eggs. After this procedure, we were able to calculate colour differences between cuckoo egg and host eggs (mimicry). For this purpose, we used the Vorobyev-Osorio receptor noise limited model of avian vision (Vorobyev \& Osorio, 1998; Vorobyev, Osorio, Bennett, Marshall, \& Cuthill, 1998) implemented in Avicol (Gomez, 2006) that reproduces bird retinal functioning and utilizes sensitivity of bird cones and nest luminosity data. In this way, we estimated chromatic contrast $(\Delta S)$ describing difference in hue. Units for $\Delta S$ are just noticeable differences (JNDs); essentially, higher values indicate higher colour difference between eggs (Gomez, 2006; Vorobyev \& Osorio, 1998; Vorobyev et al., 1998). For more details of this method, see (Šulc et al., 2016).

Spotting pattern mimicry was calculated from digital images taken by Canon Power Shot A3000 IS camera (in 2013-2016) and Canon EoS 700D with prime Canon EF 40mm lens (in 2017-2018). Photos were taken in diffuse light conditions (using a photo tent) to eliminate shadows. All photos included a grey standard (BST 14, Danes-Picta or X-Rite Colour Checker Grey Scale Chart) with known reflectance. Image linearization (in the case of Canon Power Shot camera), image calibration and pattern analysis were performed in Image (Schneider, Rasband, \& Eliceiri, 2012) using the Multispectral Image Calibration and Analysis Toolbox (Troscianko \& Stevens, 2015). All images were equally rescaled to the scale of the smallest image (11.6 pixels $/ \mathrm{mm}$ ). For pattern 
investigation we applied a granularity analysis approach filtering images with a set of spatial frequencies, and then, the pattern energy at each frequency band was measured as the standard deviation of the filtered image (Troscianko \& Stevens, 2015). Bandpass pattern analysis techniques have previously used fast Fourier transform to split the image into different spatial frequencies. While this is computationally efficient, we found that Difference-of-Gaussians (DoG) produced more effective and reliable measures, while also being a better model of neural receptive fields. Both FFT and "standard" Gaussian blur filters (such as the separable implementation in ImageJ, MATLAB, Adobe products etc...) cannot be used for non-rectangular regions-of-interest without either being affected by the background, or introducing novel pattern information, neither of which are desirable. We therefore used a custom-written Gaussian convolution filter which can be applied to an area of any region of an image without influence from its surrounds. Laplacian DoG filters were used (Malik \& Perona, 1990) where the larger Gaussian filter is 1.6 times the size of the smaller filter (we therefore used sigma values of 1 and 1.6). Convolutions such as this become computationally inefficient with large sigma values, so the image was reduced in size in order to measure the DoG at different spatial scales, building up a bandpass "energy" spectrum across a range of spatial frequencies similar to previous techniques (Troscianko \& Stevens 2015). For simplification, we calculated average pattern energy of host eggs from the particular clutch by taking the mean at each spatial scale after measuring each egg individually. Pattern energy difference between cuckoo and average host egg was calculated by summing the absolute differences in pattern energy across all spatial frequencies. As the pigments are not equally distributed on the eggshell (which can also be an important factor when recognizing parasitic eggs (Polačiková, Honza, Procházka, Topercer, \& Stokke, 2007)), we analysed pattern energy in three different parts of egg separately (sharp pole, middle part, and blunt pole) and determined pattern distribution as a standard deviation of these three pattern energies. The average pattern distribution of host eggs from the particular clutch was used for subsequent calculation of pattern distribution difference. Pattern distribution difference between cuckoo and average host egg was calculated by subtraction.

Egg width and length was measured by digital calliper with an accuracy of $0.1 \mathrm{~mm}$ and egg volume was calculated according to the formula of Hoyt (Hoyt, 1979). To obtain volume difference between cuckoo and host eggs, we compared the egg volume of the cuckoo egg with the average egg volume of host eggs from the same nest.

Finally, as we do not know which of the above traits are the most important cues for host recognition, we used a single measure of multidimensional phenotypic distance (MDPD) between cuckoo and host eggs to test the contribution of all measured differences in a single model. MDPD was defined as the Euclidean distance between two points in four-dimensional space, defined by four difference variables (chromatic contrast, pattern difference, pattern distribution difference, volume mimicry). To make the scale comparable, each of the four variables was standardized using Z-scoring (so that they all had a mean of 0 and a standard deviation of 1 ) expressing it as a proportion of its maximum 
value across all groups. For similar method, see also (Spottiswoode \& Stevens, 2011).

All calculations of cuckoo egg mimicry (colour, pattern and volume) were always performed for the cuckoo egg and only those host eggs that were present in the nest at the time of host response. All measurements were performed by single person (M.Š.) to ensure high consistency.

Data analysis

We used generalized linear mixed models with a binomial error distribution and we present full models containing all pre-planned factors of interest (Whittingham, Stephens, Bradbury, \& Freckleton, 2006). All significance values of multiple tests are based on Type III sum of squares (Crawley, 2002). In the first model, we explained the type of host rejection of cuckoo egg $(0=$ ejection, $1=$ desertion) by several independent explanatory variables describing mimicry of the cuckoo egg in the host nest, i.e. chromatic contrast, pattern difference, pattern distribution difference and volume difference and by the date in the season (laying date). As the host response can vary between years (year) and GRW female identity (individual) we put these variables into the model as random factors. We excluded the camera type we used from the random factors because we used only one type of camera within individual years, therefore this variable did not explain any variability that was not already explained by the year. In the second model, we explained the type of host rejection by the MDPD (the single measure of cuckoo egg mimicry), the laying date and their interaction and used the same random factors as in previous model (year and individual). All analyses were conducted in R 3.4.3 (R Development Core Team, 2017).

\section{Ethical note}

This study was carried out with the permission of the regional nature conservation authorities (permit numbers JMK: 115874/2013 and 38506/2016; MUHOCJ: 41433/2012/OŽP, 34437/2014/OŽP, and 14306/2016/OŽP). The fieldwork adhered to the animal care protocol (experimental project numbers 039/2011 AV ČR and 3030/ENV/17-169/630/17) and to the Czech Law on the Protection of Animals against Mistreatment (licence numbers CZ 01272 and CZ 01284).

\section{Results}

When great reed warblers face single cuckoo parasitism, they are more likely to eject the parasitic egg (62\% of 124 cases) than desert their nest. On the contrary, nest desertion was more frequent when GRW faced multiple parasitism and about $62 \%$ of nests ( $\mathrm{N}=21$ nests) were deserted.

To test whether mimicry of the cuckoo egg influences the type of host rejection, we measured mimicry in 72 of nests parasitized once (51 ejections and 21 desertions). Our first model showed that mimicry of the cuckoo egg has no effect on the type of host response. More specifically, we did not find any significant differences between cuckoo and GRW eggs in colour, pattern and volume in nests where hosts ejected cuckoo eggs compared to nests that were deserted by hosts (see Table 1 and Figure 1). We also did not find a significant 
relationship between the laying date and the type of host rejection. The second model confirmed these results; a single average variable of mimicry (MDPD) in interaction with laying date did not explain type of rejection in GRW (Table 2 and Figure 1). In a separate test we also did not find that first-time breeding females desert their nests more often than older females (three of 11 young females vs 18 of 61 old females, respectively; Fisher's exact test, $P=1$ ).

Since 12 females from our dataset were parasitized repeatedly, we assessed the consistency in their rejection type. We found that nine of them responded consistently and maintained their rejection type (two repeatedly deserted and seven repeatedly ejected). Only three females used both rejection strategies. However, it is difficult to draw firm conclusions from this small sample of repeatedly parasitized nests. Extending our dataset to include years where we did not measure mimicry (data from 2007 - 2018) showed that overall 15 repeatedly parasitized females maintained their response type (12 ejections and three desertions) and 10 females changed it which does not differ from chance $\left(\chi^{2}\right.$ test, $P=0.32$ ). Therefore, it does not seem likely that rejection type is associated with individual GRW females.
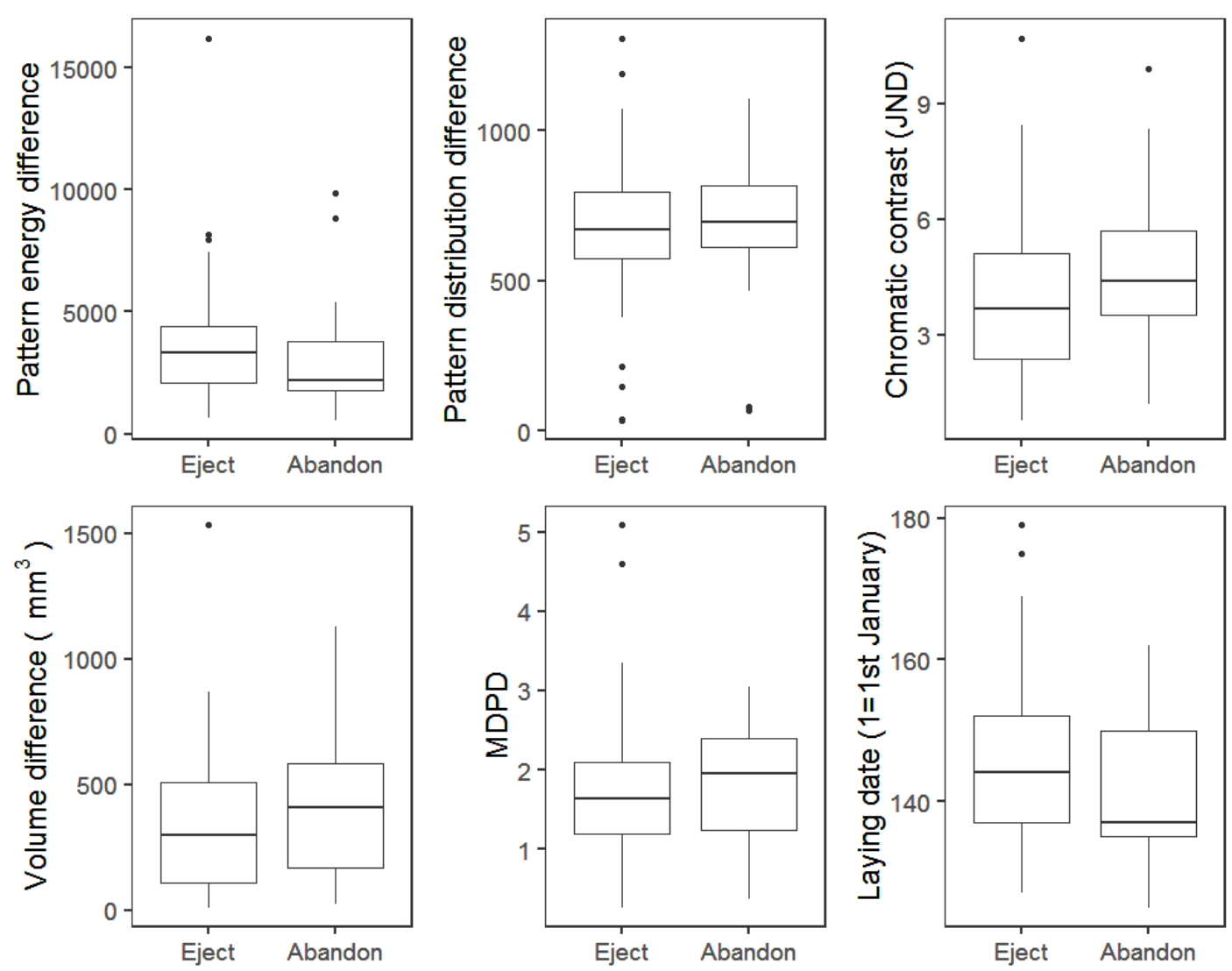

Figure 1 Cuckoo egg mimicry characteristics and laying date in naturally parasitized nests where cuckoo egg was ejected $(N=51)$ or the nest was abandoned $(N=21)$. All differences were non-significant. MDPD indicates multidimensional phenotypic distance between cuckoo and host eggs (see methods). 
Table 1 The effect of mimicry predictors and layind date on host type of response. Identity of host females and year were included as random factors.

\begin{tabular}{lcrl}
\hline \hline & & & $P$ \\
Variable & & $\boldsymbol{P}$ & \\
& & - & \\
Intercept & $-1.323902 \pm 0.736023$ & 9 & 0.0721 \\
& & - & \\
& & 0.71 & \\
Pattern energy difference & $-0.271041 \pm 0.376782$ & 9 & 0.4719 \\
Pattern distribution & & 0.01 & \\
difference & $0.005387 \pm 0.338138$ & 6 & 0.9873 \\
& & 1.04 & \\
Chromatic contrast & $0.462955 \pm 0.443275$ & 4 & 0.2963 \\
Volume difference & $0.181707 \pm 0.329382$ & 2 & 0.5812 \\
& & - & \\
Laying date & & 1.42 & \\
\hline \hline
\end{tabular}

Table 2 The effect of a single average variable of mimicry (MDPD), laying date and their interaction on host type of response. Identity of host females and year were included as random factors.

\begin{tabular}{lccc}
\hline \hline Variable & Estimate \pm SEM & $\boldsymbol{z}$ value & $\boldsymbol{P}$ value \\
\hline & $-1.30908 \pm$ & & \\
Intercept & 0.86767 & -1.509 & 0.131 \\
MDPD & 0.08855 & 0.382510 .231 & 0.817 \\
Laying date & -1.02540 & & \\
MDPD:Laying date & 0.99663 & -1.029 & 0.304 \\
\hline \hline
\end{tabular}

\section{Discussion}

Nest desertion as a defence strategy against brood parasitism is very puzzling, especially in a host that is capable of puncturing and ejecting parasitic eggs. It is clearly more beneficial for a GRW host to desert the nest than raise a cuckoo chick that kills all their progeny (Honza, Vošlajerová, \& Moskát, 2007); however, by ejecting the cuckoo egg it could achieve the same result without facing additional time and energy costs arising from building a new nest and laying new eggs.

It was proposed that desertion can be adaptive when a host realizes that its nest is parasitized but it is not able to recognize the parasitic egg reliably in its clutch and therefore cannot eject it (Hosoi \& Rothstein, 2000). Although GRW females are able to recognize and eject cuckoo eggs, the cuckoo eggs are often very good mimics (Figure 2 ), which can cause significant recognition problems, leading to a delayed response (Antonov et al., 2007; Lotem et al., 1995), less 
intense rejection (pecking) behaviour (Antonov et al., 2007) or even acceptance of parasitic eggs (Bártol et al., 2002; Moskát \& Honza, 2002). Therefore, we assumed that GRW females would benefit from nest desertion when the cuckoo egg is a good mimic of the host eggs and therefore the risk of recognition error is high (Rodriguez-Girones \& Lotem, 1999; Stokke et al., 2005). However, we found that mimicry of the cuckoo egg has no influence on the type of rejection and bad mimics were deserted even if they should theoretically be easily recognized by hosts. There are also two other studies (Higuchi, 1989; Rutila, Jokimäki, Avilés, Kaisanlahti-Jokimäki, \& Bollinger, 2006) supporting this result experimentally in the bush warbler (Cettia diphone) and common redstart (Phoenicurus phoenicurus). Therefore, we suggest that there must be other explanations for desertion behaviour than the degree of mimicry.

One possible explanation is that age affects rejection behaviour. Although it has also been suggested that young and inexperienced GRW females are worse in recognizing parasitic eggs (Lotem et al., 1992), we did not find that the young females deserted their nests more often than older ones. Therefore, age does not seem to explain the type of rejection behaviour in hosts, which is in accordance with other studies showing that age does not influence recognition abilities of hosts (Amundsen et al., 2002; Grim et al., 2014; Procházka et al., 2014; Stokke et al., 2004).

Individual variation may also affect rejection type. Individuals of some host species tend to be consistent in their response against identical model eggs and either accept or reject (Grim et al., 2014; Honza, Požgayová, Procházka, \& Tkadlec, 2007; Samaš, Hauber, Cassey, \& Grim, 2011). Interestingly, GRW females did not exhibit consistency in their type of rejection. Therefore, it does not seem likely that females simply have an innate tendency to eject or desert and instead environmental factors could affect the type of rejection.

Nest desertion can be elicited by spotting the parasitic cuckoo female at the host nest because this is potentially an important environmental stressor (Abolins-Abols \& Hauber, 2018; Soler, 2017) influencing the host response (Thomson et al., 2016). Moreover, it was demonstrated that some hosts are more likely to desert if they observe a stuffed parasite model at the nest (Davies \& Brooke, 1988; Moksnes, Røskaft, \& Korsnes, 1993; Strausberger \& Hauber, 2017). We video-recorded 15 nests from our dataset. In this sample, hosts ejected the cuckoo egg in 11 cases and deserted in four. In 12 of these nests GRW attacked the cuckoo female during parasitism and then ejected in 9 cases, deserting the other three times. In the remaining three cases we did not see the hosts while the cuckoo female was parasitizing the nest. Therefore, the visibility of the cuckoo female at the host nest does not seem to trigger desertion (Fisher's exact test, $P=1$ ). However, we cannot exclude the possibility that some host females may be more shy and inclined to desertion more easily, e.g. because of a higher sensitivity to disturbance by a parasitizing cuckoo female (Davies \& Brooke, 1988; Moksnes et al., 1993; Strausberger \& Hauber, 2017).

Another possible explanation is that a female's physical inability to eject the cuckoo egg can trigger nest desertion as was demonstrated in Acrocephalus hosts facing artificial parasitic eggs (Honza \& Moskát, 2008; Stokke et al., 2010; Šulc et al., 2015). GRWs are able to puncture and eject, however the eggshell of 
cuckoo eggs is unusually thick (Stokke, Fossøy, Røskaft, \& Moksnes, 2017), which may impose mechanical difficulties (Antonov et al., 2006) and delays in ejection (Antonov et al., 2007). Thus, if the host female has repeated problems with puncturing and ejection, she may eventually prefer to desert the nest. However, our video-recordings suggest that GRW females do not struggle with ejection of the cuckoo egg because they do not pay attention to it at all before they desert $(\mathrm{N}=4)$. Moreover, it does not seem that desertion responses are performed later than ejection by GRW females ( $N=15$ desertions, response $1.87+1.56$ days after parasitism [mean $\pm s d$ ] and $\mathrm{N}=31$ ejections, response $2.66+2.19$ days after parasitism; t.test, $\mathrm{P}=0.22$, data collected using daily nest controls) indicating that inability to eject is probably not the main factor causing desertion. Unfortunately, for precise evaluation of the puncture effort and ejection/desertion timing, continuous video-recording of more nests is needed. Moreover, nest recording also has the potential to reveal other undetected events that could have influenced our testing, e.g. multiple parasitism.

Some studies have experimentally demonstrated that desertion can be a response to a considerable reduction of clutch size (Kosciuch et al., 2006; Naomi E. Langmore, Cockburn, Russell, \& Kilner, 2009; Moskát et al., 2011; Verboven \& Tinbergen, 2002). This is probably an adaptive strategy when the number of eggs decreases below a limit where it is no longer worthwhile to continue in the given nesting attempt, because the expected future reproductive success could increase the fitness of the female significantly more (Coleman \& Gross, 1991). However, cuckoo females usually do not reduce overall clutch size during parasitism, taking mostly only one or even no host eggs (Šulc et al., 2016). This was also the case of the nests we used in this study and therefore we believe that simple egg loss did not cause nest desertion.

We have shown that a range of possible hypotheses for nest desertion do not seem to explain the patterns we have found in our data. Another possibility is that nest desertion does not always have to be costly and may be even beneficial in cases where cuckoo females removed just one host egg during parasitism. After desertion, hosts can potentially lay a complete new clutch; on the contrary, after ejection, they have a clutch reduced in size by one egg (Rothstein, 1975). This idea is supported by our results and other studies (Moskát et al., 2009; Moskát \& Honza, 2002) showing that nest desertion is more frequent in nests that were parasitized multiple times and therefore the cuckoos potentially removed more host eggs. However, it has also been shown that replacement clutches are smaller than initial ones (Klomp, 1970). Thus, rejection type may possibly depend on the condition of host females; females in good condition can afford to desert and lay a full new clutch, whereas females in worse condition eject and must settle for a reduced clutch. Future studies should investigate this question by measuring female quality and asking whether it can predict her willingness to abandon the nest.

Last, it was shown that both egg-rejection types are not randomly distributed among host taxa (Davies, 2000), e.g. most hosts of the common cuckoos (Cuculus canorus) respond by ejecting cuckoo eggs (N. E. Langmore et al., 2005; Moskát, 2005), while hosts of brown-headed cowbirds (Molothrus ater) instead abandon parasitized clutches (Hosoi \& Rothstein, 2000). Recent studies 
suggest that life history traits of both hosts and parasites, such as parasite virulence or host clutch size can explain why cuckoo hosts prefer to eject and cowbird hosts prefer to desert (Hauber et al., 2014; Servedio \& Hauber, 2006). It is possible that GRW host with its life history traits is somewhere between and both rejection strategies can persist in the host population at the same time and both can have very similar fitness consequences (Verboven \& Tinbergen, 2002).

In summary, nest desertion probably is a very effective defensive strategy against brood parasites despite the potential costs (losing time and energy for renesting and risk of being parasitized again). Although it seems that it should be costlier for hosts than the alternative strategy of ejecting the cuckoo egg from the nest, we did not find any additional factor explaining variation in these two types of host defensive behaviour. Specifically, we cannot confirm that the GRW females desert their nests because they are not able to recognize the cuckoo egg reliably in the nest. We suggest that desertion persists as a behavioural strategy because it is not as costly as previously thought and/or it is beneficial for host females in good physical condition.

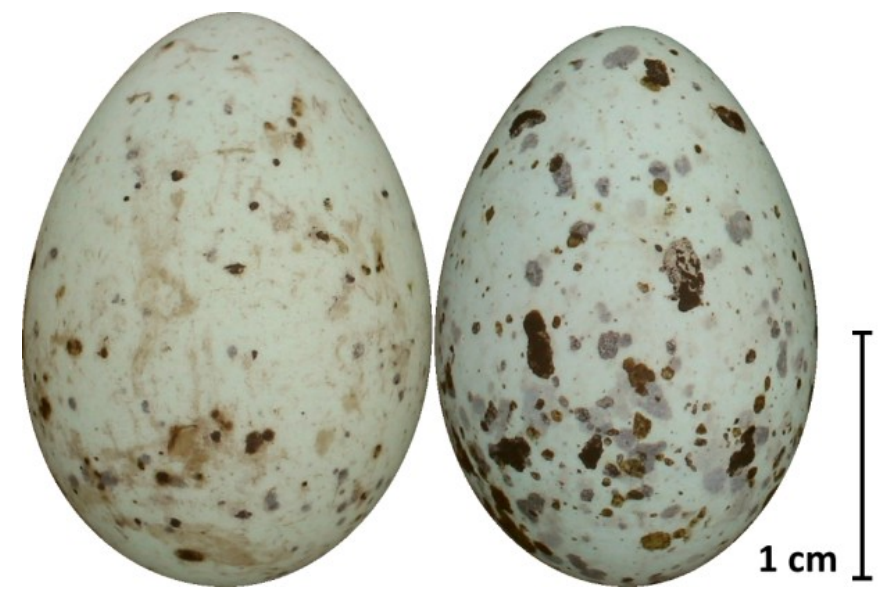

Figure 2 The cuckoo egg (left) was bigger than host GRW eggs (right) from the same clutch, but, colour, pattern energy and pattern contrast were well mimicked. Despite the very good mimicry, this cuckoo egg was recognized and ejected by hosts. 


\section{References}

Abolins-Abols, M., \& Hauber, M. E. (2018). Host defences against avian brood parasitism: an endocrine perspective. Proceedings of the Royal Society $B$ : Biological Sciences.

Amundsen, T., Brobakken, P. T., Moksnes, A., \& Røskaft, E. (2002). Rejection of common cuckoo Cuculus canorus eggs in relation to female age in the bluethroat Luscinia svecica. Journal of Avian Biology, 33(4), 366-370.

Antonov, A., Stokke, B. G., Moksnes, A., Kleven, O., Honza, M., \& Røskaft, E. (2006). Eggshell strength of an obligate brood parasite: a test of the puncture resistance hypothesis. Behavioral Ecology and Sociobiology, 60(1), 11-18.

Antonov, A., Stokke, B. G., Moksnes, A., \& Røskaft, E. (2007). Getting rid of the cuckoo Cuculus canorus egg: why do hosts delay rejection? Behavioral Ecology, 19(1), 100-107.

Arcese, P., Smith, J. N., \& Hatch, M. I. (1996). Nest predation by cowbirds and its consequences for passerine demography. Proceedings of the National Academy of Sciences, 93(10), 4608-4611.

Armstrong, T., \& Robertson, R. J. (1988). Parental investment based on clutch value: nest desertion in response to partial clutch loss in dabbling ducks. Animal Behaviour, 36(3), 941-943.

Bártol, I., Karcza, Z., Moskát, C., Røskaft, E., \& Kisbenedek, T. (2002). Responses of great reed warblers Acrocephalus arundinaceus to experimental brood parasitism: the effects of a cuckoo Cuculus canorus dummy and egg mimicry. Journal of Avian Biology, 33(4), 420-425.

Beckmann, C., \& Martin, K. (2016). Testing hypotheses about the function of repeated nest abandonment as a life history strategy in a passerine bird. Ibis, 158(2), 335-342.

Begum, S., Moksnes, A., Røskaft, E., \& Stokke, B. G. (2012). Responses of potential hosts of Asian cuckoos to experimental parasitism. Ibis, 154(2), 363-371.

Bensch, S. (1996). Female mating status and reproductive success in the great reed warbler: is there a potential cost of polygyny that requires compensation. Journal of Animal Ecology, 283-296.

Bensch, S., Hasselquist, D., Nielsen, B., \& Hansson, B. (1998). Higher fitness for philopatric than for immigrant males in a semi-isolated population of great reed warblers. Evolution, 52(3), 877-883.

Coleman, R. M., \& Gross, M. R. (1991). Parental investment theory: the role of past investment. Trends in Ecology \& Evolution, 6(12), 404-406.

Crawley, M. J. (2002). Statistical Computing: An Introduction to Data Analysis using S-Plus John Wiley and sons Chichester. UK Google Scholar.

Cuthill, I. C. (2006). Color perception. Bird Coloration, 1, 3-40.

Davies, N. B. (2000). Cuckoos, cowbirds and other cheats. T. \& AD Poyser, London.

Davies, N. B., \& Brooke, M. de L. (1988). Cuckoos versus reed warblers: adaptations and counteradaptations. Animal Behaviour, 36(1), 262-284. 
Davies, N. B., \& Brooke, M. de L. (1989). An experimental study of co-evolution between the cuckoo, Cuculus canorus, and its hosts. I. Host egg discrimination. The Journal of Animal Ecology, 207-224.

Flegeltaub, M., Biro, P. A., \& Beckmann, C. (2017). Avian nest abandonment prior to laying - a strategy to minimize predation risk? Journal of Ornithology, 158(4), 1091-1098.

Gloag, R., Fiorini, V. D., Reboreda, J. C., \& Kacelnik, A. (2013). The wages of violence: mobbing by mockingbirds as a frontline defence against broodparasitic cowbirds. Animal Behaviour, 86(5), 1023-1029.

Goguen, C. B., \& Mathews, N. E. (1996). Nest desertion by blue-gray gnatcatchers in association with brown-headed cowbird parasitism. Animal Behaviour, 52(3), 613-619.

Gomez, D. (2006). AVICOL, a program to analyse spectrometric data. Available from the Author upon Request at Dodogomez@ Yahoo.Fr.

Grim, T., Samaš, P., \& Hauber, M. E. (2014). The repeatability of avian egg ejection behaviors across different temporal scales, breeding stages, female ages and experiences. Behavioral Ecology and Sociobiology, 68(5), 749-759.

Guigueno, M. F., \& Sealy, S. G. (2010). Clutch abandonment by parasitized yellow warblers: egg burial or nest desertion? The Condor, 112(2), 399-406.

Hanley, D., Samaš, P., Hauber, M. E., \& Grim, T. (2015). Who moved my eggs? An experimental test of the egg arrangement hypothesis for the rejection of brood parasitic eggs. Animal Cognition, 18(1), 299-305.

Hanley, D., Šulc, M., Brennan, P. L., Hauber, M. E., Grim, T., \& Honza, M. (2016). Dynamic egg color mimicry. Ecology and Evolution, 6(12), 4192-4202.

Hauber, M. E., Samaš, P., Anderson, M. G., Rutila, J., Low, J., Cassey, P., \& Grim, T. (2014). Life-history theory predicts host behavioural responses to experimental brood parasitism. Ethology Ecology \& Evolution, 26(4), 349364.

Higuchi, H. (1989). Responses of the bush warbler Cettia diphone to artificial eggs of Cuculus cuckoos in Japan. Ibis, 131(1), 94-98.

Hill, D. P., \& Sealy, S. G. (1994). Desertion of nests parasitized by cowbirds: Have Clay-coloured Sparrows evolved an anti-parasite defence? Animal Behaviour, 48(5), 1063-1070.

Honza, M., \& Moskát, C. (2008). Egg rejection behaviour in the great reed warbler (Acrocephalus arundinaceus): the effect of egg type. Journal of Ethology, 26(3), 389.

Honza, M., Požgayová, M., Procházka, P., \& Tkadlec, E. (2007). Consistency in egg rejection behaviour: responses to repeated brood parasitism in the blackcap (Sylvia atricapilla). Ethology, 113(4), 344-351.

Honza, M., Vošlajerová, K., \& Moskát, C. (2007). Eviction behaviour of the common cuckoo Cuculus canorus chicks. Journal of Avian Biology, 38(3), 385-389.

Hosoi, S. A., \& Rothstein, S. I. (2000). Nest desertion and cowbird parasitism: evidence for evolved responses and evolutionary lag. Animal Behaviour, 59(4), 823-840. 
Hoyt, D. F. (1979). Practical methods of estimating volume and fresh weight of bird eggs. The Auk, 73-77.

Immelmann, K. (1971). Ecological aspects of periodic reproduction. Avian Biology, 1, 341-389.

Jelínek, V., Procházka, P., \& Honza, M. (2015). Experimental enlargement of nest size does not increase risk of predation or brood parasitism in the Great Reed Warbler Acrocephalus arundinaceus. Ibis, 157(2), 396-400.

Jennions, M. D., \& Polakow, D. A. (2001). The effect of partial brood loss on male desertion in a cichlid fish: an experimental test. Behavioral Ecology, 12(1), 84-92.

Klomp, H. (1970). The determination of clutch-size in birds a review. Ardea, 55(12), 1-125.

Kosciuch, K. L., Parker, T. H., \& Sandercock, B. K. (2006). Nest desertion by a cowbird host: an antiparasite behavior or a response to egg loss? Behavioral Ecology, 17(6), 917-924.

Langmore, N. E., Kilner, R. M., Butchart, S. H. M., Maurer, G., Davies, N. B., Cockburn, A., ... Dowling, D. K. (2005). The evolution of egg rejection by cuckoo hosts in Australia and Europe. Behavioral Ecology, 16(4), 686-692.

Langmore, Naomi E., Cockburn, A., Russell, A. F., \& Kilner, R. M. (2009). Flexible cuckoo chick-rejection rules in the superb fairy-wren. Behavioral Ecology, 20(5), 978-984.

Lotem, A., Nakamura, H., \& Zahavi, A. (1992). Rejection of cuckoo eggs in relation to host age: a possible evolutionary equilibrium. Behavioral Ecology, 3(2), 128-132.

Lotem, A., Nakamura, H., \& Zahavi, A. (1995). Constraints on egg discrimination and cuckoo-host co-evolution. Animal Behaviour, 49(5), 1185-1209.

Malik, J., \& Perona, P. (1990). Preattentive texture discrimination with early vision mechanisms. JOSA A, 7(5), 923-932.

Martín-Vivaldi, M., Soler, J. J., Møller, A. P., Pérez-Contreras, T., \& Soler, M. (2013). The importance of nest-site and habitat in egg recognition ability of potential hosts of the C ommon C uckoo C uculus canorus. Ibis, 155(1), 140-155.

Moksnes, A., Røskaft, E., \& Braa, A. T. (1991). Rejection behavior by common cuckoo hosts towards artificial brood parasite eggs. The Auk, 348-354.

Moksnes, A., Røskaft, E., \& Korsnes, L. (1993). Rejection of cuckoo (Cuculus canorus) eggs by meadow pipits (Anthus pratensis). Behavioral Ecology, $4(2), 120-127$.

Molnár, B. (1944). The cuckoo in the Hungarian plain. Aquila, 51, 100-112.

Moskát, C. (2005). Common Cuckoo parasitism in Europe: behavioural adaptations, arms race and the role of metapopulations. Ornithological Science, 4(1), 3-15.

Moskát, C., Hauber, M. E., Avilés, J. M., Bán, M., Hargitai, R., \& Honza, M. (2009). Increased host tolerance of multiple cuckoo eggs leads to higher fledging success of the brood parasite. Animal Behaviour, 77(5), 1281-1290.

Moskát, C., \& Honza, M. (2002). European cuckoo Cuculus canorus parasitism and host's rejection behaviour in a heavily parasitized great reed warbler Acrocephalus arundinaceus population. Ibis, 144(4), 614-622. 
Moskát, C., Rosendaal, E. C., Boers, M., Zölei, A., Bán, M., \& Komdeur, J. (2011). Post-ejection nest-desertion of common cuckoo hosts: a second defense mechanism or avoiding reduced reproductive success? Behavioral Ecology and Sociobiology, 65(5), 1045-1053.

Øien, I. J., Moksnes, A., Røskaft, E., \& Honza, M. (1998). Costs of cuckoo Cuculus canorus parasitism to reed warblers Acrocephalus scirpaceus. Journal of Avian Biology, 209-215.

Peer, B. D., \& Rothstein, S. I. (2010). Phenotypic plasticity in common grackles (Quiscalus quiscula) in response to repeated brood parasitism. The Auk, 127(2), 293-299.

Polačiková, L., Honza, M., Procházka, P., Topercer, J., \& Stokke, B. G. (2007). Colour characteristics of the blunt egg pole: cues for recognition of parasitic eggs as revealed by reflectance spectrophotometry. Animal Behaviour, 74(3), 419-427.

Požgayová, M., Procházka, P., \& Honza, M. (2009). Sex-specific defence behaviour against brood parasitism in a host with female-only incubation. Behavioural Processes, 81(1), 34-38.

Procházka, P., Jelínek, V., Požgayová, M., \& Honza, M. (2012). How to age great reed warblers (Acrocephalus arundinaceus) after complete moult. Sylvia, 48, 57-73.

Procházka, P., Konvičková-Patzenhauerová, H., Požgayová, M., Trnka, A., Jelínek, V., \& Honza, M. (2014). Host genotype and age have no effect on rejection of parasitic eggs. Naturwissenschaften, 101(5), 417-426.

R Development Core Team, R. (2017). R: A language and environment for statistical computing. $\mathrm{R}$ foundation for statistical computing Vienna, Austria.

Ratcliff, R., Smith, P. L., Brown, S. D., \& McKoon, G. (2016). Diffusion decision model: Current issues and history. Trends in Cognitive Sciences, 20(4), 260-281.

Roche, E. A., Arnold, T. W., \& Cuthbert, F. J. (2010). Apparent nest abandonment as evidence of breeding-season mortality in Great Lakes Piping Plovers (Charadrius melodus). The Auk, 127(2), 402-410.

Rodriguez-Girones, M. A., \& Lotem, A. (1999). How to detect a cuckoo egg: a signal-detection theory model for recognition and learning. The American Naturalist, 153(6), 633-648.

Rohwer, S., \& Spaw, C. D. (1988). Evolutionary lag versus bill-size constraints: a comparative study of the acceptance of cowbird eggs by old hosts. Evolutionary Ecology, 2(1), 27-36.

Rothstein, S. I. (1975). An experimental and teleonomic investigation of avian brood parasitism. The Condor, 77(3), 250-271.

Rutila, J., Jokimäki, J., Avilés, J. M., Kaisanlahti-Jokimäki, M.-L., \& Bollinger, E. K. (2006). Responses of Parasitized And Unparasitized Common Redstart (Phoenicurus Phoenicurus) Populations Against Artificial Cuckoo Parasitism. The Auk, 123(1), 259-265.

Samaš, P., Hauber, M. E., Cassey, P., \& Grim, T. (2011). Repeatability of foreign egg rejection: testing the assumptions of co-evolutionary theory. Ethology, $117(7), 606-619$. 
Schneider, C. A., Rasband, W. S., \& Eliceiri, K. W. (2012). NIH Image to ImageJ: 25 years of image analysis. Nature Methods, 9(7), 671.

Sealy, S. G. (1995). Burial of cowbird eggs by parasitized yellow warblers: an empirical and experimental study. Animal Behaviour, 49(4), 877-889.

Sealy, S. G., \& Lorenzana, J. C. (1997). Yellow Warblers (Dendroica petechia) do not recognize their own eggs. Bird Behavior, 12(3-4), 57-66.

Servedio, M. R., \& Hauber, M. E. (2006). To eject or to abandon? Life history traits of hosts and parasites interact to influence the fitness payoffs of alternative anti-parasite strategies. Journal of Evolutionary Biology, 19(5), 1585-1594.

Soler, M. (1990). Relationships between the great spotted cuckoo Clamator glandarius and its corvid hosts in a recently colonized area. Ornis Scandinavica, 212-223.

Soler, M. (2017). Brood Parasitism in Birds: A Coevolutionary Point of View. In Avian Brood Parasitism (pp. 1-19). Springer.

Soler, M., Ruiz-Raya, F., Roncalli, G., \& Ibáñez-Álamo, J. D. (2015). Nest desertion cannot be considered an egg-rejection mechanism in a medium-sized host: an experimental study with the common blackbird Turdus merula. Journal of Avian Biology, 46(4), 369-377.

Spottiswoode, C. N., \& Stevens, M. (2011). How to evade a coevolving brood parasite: egg discrimination versus egg variability as host defences. Proceedings of the Royal Society of London B: Biological Sciences, rspb20110401.

Stokke, B. G., Fossøy, F., Røskaft, E., \& Moksnes, A. (2017). Adaptations of Brood Parasitic Eggs. In Avian Brood Parasitism (pp. 363-384). Springer.

Stokke, B. G., Moksnes, A., \& Røskaft, E. (2005). The enigma of imperfect adaptations in hosts of avian brood parasites. Ornithological Science, 4(1), 17-29.

Stokke, B. G., Polačiková, L., Dyrcz, A., Hafstad, I., Moksnes, A., \& Røskaft, E. (2010). Responses of Reed Warblers Acrocephalus scirpaceus to nonmimetic eggs of different sizes in a nest parasitism experiment. Acta Ornithologica, 45(1), 98-104.

Stokke, B. G., Rudolfsen, G., Moksnes, A., \& Røskaft, E. (2004). Rejection of conspecific eggs in chaffinches: the effect of age and clutch characteristics. Ethology, 110(6), 459-470.

Strausberger, B. M., \& Hauber, M. E. (2017). Encounters With Adult Brood Parasitic Cowbirds At Nests, Not Costly Parasitic Eggs, Elicit Nest Desertion By Chipping Sparrows (Spizella passerina). The Wilson Journal of Ornithology, 129(3), 554-559.

Šulc, M., Procházka, P., Capek, M., \& Honza, M. (2015). Birds use eggshell UV reflectance when recognizing non-mimetic parasitic eggs. Behavioral Ecology, 27(2), 677-684.

Šulc, M., Procházka, P., Capek, M., \& Honza, M. (2016). Common cuckoo females are not choosy when removing an egg during parasitism. Behavioral Ecology, 27(6), 1642-1649. https://doi.org/10.1093/beheco/arw085

Thomson, R. L., Tolvanen, J., \& Forsman, J. T. (2016). Cuckoo parasitism in a cavity nesting host: near absent egg-rejection in a northern redstart 
population under heavy apparent (but low effective) brood parasitism. Journal of Avian Biology, 47(3), 363-370.

Troscianko, J., \& Stevens, M. (2015). Image calibration and analysis toolbox-a free software suite for objectively measuring reflectance, colour and pattern. Methods in Ecology and Evolution, 6(11), 1320-1331.

Verboven, N., \& Tinbergen, J. M. (2002). Nest desertion: a trade-off between current and future reproduction. Animal Behaviour, 63(5), 951-958.

Vorobyev, M., \& Osorio, D. (1998). Receptor noise as a determinant of colour thresholds. Proceedings of the Royal Society of London B: Biological Sciences, 265(1394), 351-358.

Vorobyev, M., Osorio, D., Bennett, A. T., Marshall, N. J., \& Cuthill, I. C. (1998). Tetrachromacy, oil droplets and bird plumage colours. Journal of Comparative Physiology A, 183(5), 621-633.

Whittingham, M. J., Stephens, P. A., Bradbury, R. B., \& Freckleton, R. P. (2006). Why do we still use stepwise modelling in ecology and behaviour? Journal of Animal Ecology, 75(5), 1182-1189.

Zink, A. G. (2003). Quantifying the costs and benefits of parental care in female treehoppers. Behavioral Ecology, 14(5), 687-693.

Zuckerman, Z. C., Philipp, D. P., \& Suski, C. D. (2014). The influence of brood loss on nest abandonment decisions in largemouth bass Micropterus salmoides. Journal of Fish Biology, 84(6), 1863-1875. 